\title{
Vorhersage von Psychosen durch stufenweise Mehrebenenabklärung - Das Basler FePsy (Früherkennung von Psychosen)-Projekt
}

Anita Riecher-Rössler ${ }^{1 *}$, Jacqueline Aston ${ }^{1}$, Stefan Borgwardt ${ }^{2,3}$, Hilal Bugra ${ }^{1}$, Peter

Fuhr $^{4}$, Ute Gschwandtner ${ }^{4}$, Nikolaos Koutsouleris ${ }^{5}$, Marlon Pflüger ${ }^{6}$, Corinne Tamagni ${ }^{1}$, Ernst-Wilhelm Radü ${ }^{3}$, Charlotte Rapp ${ }^{1}$, Renata Smieskova ${ }^{2,3}$, Erich Studerus ${ }^{1}$, Anna Walter ${ }^{1}$, Ronan Zimmermann ${ }^{4}$

${ }^{1}$ Zentrum für Gender Research und Früherkennung, Universitäre Psychiatrische Kliniken Basel, Universität Basel

${ }^{2}$ Zentrum für Diagnostik und Krisenintervention, Universitäre Psychiatrische Kliniken Basel, Universität Basel

${ }^{3}$ Medical Image Analysis Center (MIAC), Universitätsspital Basel, Basel, Schweiz

${ }^{4}$ Neurologische Klinik, Universitätsspital Basel, Basel, Schweiz

${ }^{5}$ Klinik für Psychiatrie und Psychotherapie, Ludwig-Maximilians-Universität, München, Deutschland

${ }^{6}$ Forensisch-Psychiatrische Klinik, Universitäre Psychiatrische Kliniken Basel, Basel, Schweiz

\section{*Korrespondenzadresse:}

Prof. Dr. med. Anita Riecher-Rössler

Chefärztin und Ordinaria

Universitäre Psychiatrische Kliniken Basel

Zentrum für Gender Research und Früherkennung

c/o Universitätsspital Basel

Petersgraben 4

$\mathrm{CH}-4031$ Basel

Tel.: +41612655114

Fax: +4161265 4599

Email: Anita.Riecher@upkbs.ch 


\section{ZUSAMMENFASSUNG}

Hintergrund: In Basel haben wir verschiedene Studien zur Verbesserung der Methodik zur Früherkennung von Psychosen (FePsy) durchgeführt.

Methodik: Vom 01.03.2000 bis 29.02.2004 wurden 234 Individuen mithilfe des Basler Screening Instruments für Psychosen (BSIP) gescreent. Bei 106 Patienten konnte ein Risikostatus für Psychosen diagnostiziert werden, 53 davon konnten bis zu 7 Jahre (Mittel 5.4 Jahre) nachuntersucht werden.

Die weiteren Untersuchungen erfolgten u.a. mit einem spezifisch entwickelten Anamnese-Instrument, verschiedenen Skalen zur Psychopathologie, Untersuchungen der Neuropsychologie und Feinmotorik, klinischem und quantitativem EEG, MRI des Gehirns, Labor.

Ergebnisse: Allein auf der Basis des BSIP konnte eine relativ zuverlässige Vorhersage getroffen werden: 21 (39.6\%) der als „Risikopatienten“ Identifizierten entwickelten innerhalb der Beobachtungszeit tatsächlich eine Psychose. Post hoc konnte durch spezifischere Gewichtung der Psychopathologie und Einbezug neuropsychologischer Untersuchungen die Vorhersagegenauigkeit auf $81 \%$ gesteigert werden. Die anderen oben genannten Verfahren können offensichtlich zur weiteren Verbesserung der Prädiktion beitragen.

Schlussfolgerungen: Die Risikoabklärung für Psychose sollte stufenweise und unter Einbezug verschiedener Untersuchungsebenen erfolgen.

Schlüsselwörter: Psychose, Risiko, Prädiktion, Neuropsychologie, Feinmotorik, EEG, MRI 


\section{ABSTRACT}

Background: We have conducted various studies in Basel with the aim of improving the methods for the early detection of psychosis (Früherkennung von Psychosen, FePsy).

Methods: From 01.03.2000 to 29.02.2004 234 individuals were screened using the Basel Screening Instrument for Psychosis (BSIP). 106 patients were identified as at risk for psychosis; out of these 53 remained in follow-up for up to 7 years (mean 5.4 years).

The assessments were done with a specifically developed instrument for history taking, various scales for the psychopathology, assessments of neuropsychology and fine motor functioning, clinical and quantitative EEG, MRI of the brain, laboratory etc. Results: Based on the BSIP alone, a relatively reliable prediction was possible: 21 $(39.6 \%)$ of the individuals identified as at risk developed psychosis within the followup time.

Post-hoc prediction could be improved to $81 \%$ by weighting psychopathology and including neuropsychology. Including the other domains obviously allows further improvements of prediction.

Conclusions: The risk for psychosis should be assessed in a stepwise procedure. In a first step a clinically oriented screening should be conducted. If an at-risk status is found, further assessments in various domains should be done in a specialised center.

Keywords: Psychosis, At Risk Mental State, prediction, neuropsychology, fine motor functioning, EEG, MRI 


\section{TAKE-HOME-MESSAGE}

- Eine beginnende Psychose kann schon in ihrem präpsychotischen Prodromalstadium relativ zuverlässig erkannt werden.

- Die von uns entwickelten Instrumente „Risiko-Checkliste“, „SelbstscreenProdrom" sowie das Screening-Instrument für Psychosen (BSIP) können hier hilfreich sein.

- Wird so ein Risikostatus identifiziert, sollten weitere Abklärungen auf verschiedenen Untersuchungsebenen in einem hierfür spezialisierten Zentrum erfolgen. 


\section{HINTERGRUND}

Die Früherkennung und Frühbehandlung von Psychosen ist in den letzten 10 Jahren zu einem weithin akzeptierten Ziel der Psychiatrie geworden. Spezialisierte Früherkennungs- und Frühbehandlungszentren wurden weltweit etabliert. Ausgelöst wurde diese Entwicklung durch verschiedene Beobachtungen und Erkenntnisse:

- Schizophrene Psychosen beginnen meist schleichend und atypisch.

- Die Diagnose und Behandlung dieser Psychosen erfolgte bisher häufig nur mit grosser Verzögerung.

- Ernste Folgen sind schon in oft noch undiagnostizierten Frühphasen, ja sogar in der noch unspezifischen sogenannten Prodromalphase beobachtbar.

- Eine frühe Behandlung kann Verlauf und Prognose entscheidend verbessern. (Übersichten bei: [1-4]).

$\mathrm{Zu}$ Beginn fokussierten die Früherkennungs- und Frühbehandlungszentren auf Patienten mit klaren psychotischen Ersterkrankungen. Nachdem jedoch erkannt wurde, welche negativen Folgen die beginnende Erkrankung schon davor, nämlich in ihren präpsychotischen Prodromalstadien haben kann, wurde versucht, die beginnende Ersterkrankung auch schon früher zu erfassen, d.h. Individuen mit einer Prodromalsymptomatik oder einem sogenannten klinischen Risikostatus (At Risk Mental State: ARMS) zu identifizieren [5].

Die Identifikation eines Prodromalstadiums ist jedoch äusserst schwierig, zumal versucht werden muss, möglichst wenig Patienten mit einem echten Prodromalzustand zu "verpassen", gleichzeitig aber möglichst wenige fälschlich als Risikopatienten einzustufen [6]. Zahlreiche Forschungsgruppen versuchen deshalb, die Methodik der Frühidentifikation zu verbessern. In ersten klinischen Prädiktionsstudien konnte eine Vorhersagegenauigkeit für Transitionen von $9-54 \%$ erreicht werden [2,7-9]). Eine kürzlich erschienene Meta-Analyse zu diesem Thema berichtete eine konsistente Transitionsrate von 18\% nach 6 Monaten Follow-up, 22\% nach einem Jahr, 29\% nach 2 Jahren und 36\% nach 3 Jahren [10]. Eine KatamneseStudie von Klosterkötter et al. [11], die einen 10-Jahres-Zeitraum abdeckte, legt sogar eine $70 \%$ Transitionsrate nahe. Je länger also der Beobachtungszeitraum ist, desto mehr der Risikoindividuen entwickeln offensichtlich eine Psychose. Trotz 
dieser vergleichsweise guten Vorhersagegenauigkeit wurde immer wieder kritisiert, auf der Basis der gegenwärtigen Abklärungen und Einschlusskriterien würden zu viele sogenannte Falschpositive identifiziert, Patienten also, die unnötigerweise mit einem Risikostatus konfrontiert würden. Andererseits würden viele Patienten mit einem Risikostatus unentdeckt bleiben, wenn die Kriterien nicht sensitiv genug wären. Um dieses Dilemma zu lösen, ist eine Verbesserung der Zuverlässigkeit der Risikoabschätzung dringend notwendig.

Während in älteren Studien vor allem die Psychopathologie, das genetische Risiko und sozialer Abstieg als Risikokriterien dienten, haben in den letzten Jahren zunehmend auch andere Untersuchungsebenen, wie wir sie 2006 in einem Übersichtsartikel zusammengestellt haben [1], an Bedeutung gewonnen, wie etwa die Neuropsychologie, das EEG oder die zerebrale Bildgebung. So haben verschiedene Autoren Prädiktionsmodelle vorgelegt unter Einbeziehung spezifischer Psychopathologie [z.B. 8,9], der Neuropsychologie [z.B. 2,12,13], des Neuroimaging [z.B. 14,15,16], des quantitativen EEG (Q-EEG) [z.B. 17,18] oder evozierter Potentiale $[19,20]$.

Ziel der Basler Früherkennung von Psychosen (FePsy) Studien ist die Verbesserung der Methodik zur Einschätzung des Risikostatus (At Risk Mental State: ARMS) bzw. zur Prädiktion des Übergangs in eine Psychose auf der Basis eines gestuften Vorgehens, d.h. eines Screenings in einem ersten Schritt und bei entsprechendem, auf dieser Basis identifiziertem Risiko, einer Mehrebenenabklärung.

Unsere Hypothesen:

1. Die Identifikation von Risiko-Individuen ist möglich in einem stufenweisen Prozess mit einem klinischen Screening als erstem Schritt.

2. Die Identifikation von Risiko-Individuen kann verbessert werden durch a) Post-hoc-Vergleiche zwischen den Risiko-Individuen mit und ohne späteren Übergang in eine Psychose und b) umfassende Multiebenen-Untersuchungen. 


\section{StichPROBE UNd Methoden}

\section{Studiendesign}

Die Früherkennung von Psychosen (FePsy) Studie ist eine offene, prospektive klinische Studie aller konsekutiven Aufnahmen in eine Spezialsprechstunde zur Früherkennung von Psychosen [21]. Vom 1. März 2000 bis zum 29. Februar 2004 wurden in einer ersten Projektphase Individuen mit einem vermutlichen Risikostatus auf der Basis des Basler Screening Instrumentes für Psychosen (BSIP) [22] identifiziert und, wenn sie ein Risiko zeigten, genauer untersucht unter Einbeziehung der verschiedensten Ebenen wie spezifische Anamneseerhebung unter Berücksichtigung aller bekannten Risikofaktoren für Psychosen, Psychopathologie, Neuropsychologie, EEG, Neuroimaging, Labor. Alle Risikopersonen wurden daraufhin regelmässig nachuntersucht. In einer Post-hoc-Analyse nach 7 Jahren wurden sodann diejenigen Patienten, die inzwischen tatsächlich eine manifeste Psychose entwickelt hatten mit denjenigen verglichen, die nicht erkrankt waren, um so die Prädiktion der Transition noch weiter zu verbessern.

\section{Setting}

Die Patienten wurden über die FePsy-Sprechstunde an der Psychiatrischen Universitätspoliklinik Basel, Schweiz, rekrutiert. Um eine möglichst frühe, niederschwellige Überweisung solcher Patienten an die Sprechstunde zu erreichen, führten wir zu Beginn und dann immer wieder breite Informationskampagnen für Laien und Professionelle durch [21].

Im Rahmen dieser Informationskampagnen wurde an potentielle Zuweiser eine sogenannte Risiko-Checkliste verteilt (Abb. 1) sowie ein Selbstbeurteilungsbogen zur Abgabe an potentiell Betroffene (Abb. 2) [23]. Beide Instrumente hatten wir speziell für diesen Zweck entwickelt, ebenso wie die Website des FePsy-Programms (http://www.fepsy.ch/).

Alle überwiesenen Patienten wurden sodann mithilfe des Basel Screening Instruments für Psychosen (BSIP) [22] gescreent (siehe unten). 
- Abb. 1 und Abb. 2 bitte etwa hier einfügen -

\section{INSTRUMENTE UND MESSMETHODEN}

\section{Screening-Instrument und Einschlusskriterien}

Der Risikostatus wurde auf der Basis des Basel Screening Instruments für Psychosen (BSIP), das speziell für diesen Zweck entwickelt worden war, eingeschätzt (Tab. 1a) $[21,22]$. Dieses Instrument basiert auf den DSM-IIIProdromalsymptomen [24] und anderen aus der Literatur exzerpierten Prodromen, sowie Risikofaktoren und Indikatoren wie sozialer Abstieg, Drogenabusus, frühere psychiatrische Erkrankung und genetisches Risiko. Vier Psychose-Items wurden aus der Brief Psychiatric Rating Scale (BPRS) $[25,26]$ übernommen, um die präpsychotischen Phänomene zu beurteilen. Das BSIP erlaubt die Einschätzung der Individuen auf Basis der Ein- und Ausschlusskriterien entsprechend den Melbourner PACE-Kriterien zur Einschätzung des Risikostatus (Tab. 1b) [5,27]. Das BSIP hat eine gute Interrater-Reliabilität $(k=.67)$ für die Einschätzung des Psychose-Risikos sowie eine hohe prädiktive Validität [22].

- Tab. 1a und Tab. $1 b$ bitte etwa hier einfügen -

\section{Eingangsuntersuchung}

Alle Individuen, die aufgrund des Screenings die Einschlusskriterien für die Studie bzw. den Risikostatus für Psychose erfüllten, wurden umfangreichen Untersuchungen unterzogen, einschliesslich Brief Psychiatric Scale (BPRS) [26], Scale for the Assessment of Negative Symptoms (SANS) [28] und anderen Psychopathologie-Instrumenten, einem ausführlichen Anamneseinstrument sowie einer neuropsychologischen Testbatterie [29-35], Feinmotorik-Untersuchungen [36], klinischem und quantitativem EEG [17,18], MRI des Gehirns [37,38] und Labor [39].

\section{Follow-up}

Jede Risikoperson wurde regelmässig nachuntersucht, im ersten Jahr monatlich, im zweiten und dritten Jahr dreimonatlich und danach jährlich. Der Übergang in eine 
Psychose wurde dabei mit Hilfe des BPRS [26] und der Transitionskriterien nach Yung et al. [5] erfasst (Tab. 1b). Die Patienten und deren Behandler (meist in unserer Poliklinik) wurden angehalten, sich auch zwischen den regelmässigen Follow-upTerminen zu melden, wenn die Symptome sich verschlechterten.

\section{Stichprobe}

234 Patienten wurden in diesen ersten vier Jahren des Projekts gescreent. Von diesen erfüllten 106 die Kriterien für einen Risikostatus, 64 davon nahmen an unserer Studie teil (Details bei [2,21]). Es gab keine signifikanten Unterschiede zwischen Teilnehmern und Nichtteilnehmern im Hinblick auf wesentliche Merkmale wie Alter oder Geschlecht. Während des Follow-ups zogen neun Risikopersonen ihre Teilnahme zurück. Zwei weitere Patienten wurden ausgeschlossen, weil sie durch klinik-externe Psychiater hochpotente Neuroleptika wegen zunehmender Minussymptomatik erhielten. Das heisst, 53 ARMS-Individuen [40], 21\% Frauen und $32 \%$ Männer, verblieben im Follow-up, entweder bis zum Übergang in eine Psychose oder bis zum Endpunkt unserer Analyse (28.02.2007).

Es gab keine signifikanten Unterschiede zwischen den 53 ARMS-Individuen, die im Follow-up verblieben, und den 11 „Dropouts“, die wir im Laufe der Studie verloren, im Hinblick auf wesentliche Merkmale wie Alter, Geschlecht, Bildung, Dauer der unbehandelten Erkrankung, Einschlusskriterien, Medikation, Cannabis-Abusus, BPRS- oder SANS-Gesamtscore.

Fast 90\% der 53 ARMS-Individuen, die im Follow-up verblieben, waren aufgrund präpsychotischer (v.a. attenuierter, also unterschwelliger psychotischer) Symptome eingeschlossen worden oder auf der Basis einer Kombination solcher Symptome mit einem genetischen Risiko. Nur zwei Individuen waren v.a. aufgrund ihres genetischen Risikos eingeschlossen worden und vier aufgrund einer Kombination unspezifischer Risikofaktoren (für Details siehe [2,21]). 


\section{Statistische Methoden}

SPSS für Windows (Version 15) wurde verwendet; für die kategorialen Variablen $x^{2}$ oder Fisher's exact tests, für intervall- oder ordinal-skalierte Variablen Mann-Whitney $\mathrm{U}$ - oder Student t-Tests. Bei multiplen Testungen wurden Bonferroni-Korrekturen durchgeführt. Zum Teil wurden multivariate Varianzanalysen (MANOVA) durchgeführt. Die Zeit bis zur Psychose-Transition wurde mit der Kaplan-Meier Methode für zensierte Daten analysiert. Für die Analyse neuropsychologischer Daten wurden umfangreiche Vorprozessierungen durchgeführt (siehe [41]). Für das Prädiktormodell unter Einbezug psychopathologischer und neuropsychologischer Variablen wurden die Variablen mit Hilfe einer schrittweisen logistischen Regression mit Rückwärtseliminations-Algorithmus ausgewählt (für Details siehe [2]). Die Cut-offScores, um die Sensitivität, Spezifität und Vorhersagegenauigkeit zu bestimmen, wurden von der „Receiver Operating Characteristic curve“ [42] abgeleitet.

Für die Analyse des EEG, Q-EEG und Neuroimaging bedienten wir uns spezieller statistischer Verfahren, die in den jeweiligen Publikationen (siehe unten) genauer beschrieben sind.

\section{ERGEBNISSE}

\section{1. Übergang in Psychose}

21 (39.6\%) der 53 Risiko-Individuen, die im Follow-up verblieben waren, entwickelten tatsächlich eine eindeutige Psychose entsprechend der Kriterien von Yung et al. [5]. Die kumulative Übergangsrate nach 7 Jahren betrug .34 (Kaplan-Meier-Schätzung). Die meisten Psychosen entwickelten sich während der ersten 12 Monate der Beobachtung, 29\% aber noch danach (Tab. 2). Die mittlere Zeit bis zum Übergang in eine klare Psychose betrug 11.6 Monate $(95 \% \mathrm{KI}=5.7-17.5$ Monate, $\mathrm{SD}=13$ Monate, Median = 10 Monate). Nur drei Individuen konvertierten ultra-rasch, nämlich innerhalb der ersten vier Beobachtungswochen, ein Individuum aber noch nach 55 Monaten. Auch die Patienten ohne Übergang in eine Psychose wurden im Mittel 5.4 
Jahre lang nachbeobachtet (Median 66 Monate, Minimum 37 Monate, Maximum 82 Monate).

- Tab. 2 bitte etwa hier einfügen -

\section{Vergleich der Patienten mit versus ohne Übergang in Psychose}

In einer Post-hoc-Analyse verglichen wir sodann die ARMS-Patienten mit Transition in eine Psychose innerhalb der Beobachtungszeit (ARMS-T), mit denen ohne Transition, Non-Transition (ARMS-NT), auf der Basis ihrer Charakteristika, die sie bei Einschluss in die Studie geboten hatten. Wir versuchten dadurch, innerhalb der von uns identifizierten Risikopopulation noch spezifischere Prädiktoren des tatsächlichen Übergangs in eine Psychose zu bestimmen.

\subsection{Demographische und klinische Charakteristika}

In Bezug auf die meisten demographischen und klinischen Charakteristika zeigten sich keine Unterschiede zwischen den beiden Patientengruppen, auch nicht bezüglich ihrer Risikofaktoren, ihrer Prodromalsymptome oder anderer Indikatoren einer beginnenden Psychose. Jedoch waren die Patienten mit späterer Transition (ARMS-T), also die ,echten' Prodromal-Patienten, häufiger als andere Patienten wegen präpsychotischer Symptome, v.a. attenuierter, unterschwelliger psychotischer Symptome, eingeschlossen worden (für Details siehe [2]).

Auch hatten die ,echten' Prodromal-Patienten schon bei Aufnahme signifikant mehr Misstrauen und eine Tendenz für einen höheren Gesamtscore im BPRS gezeigt (Tab. 3). Des Weiteren hatten sie schon bei Einschluss einen höheren SANSGesamtscore, also mehr Minus-Symptomatik gezeigt, und hier insbesondere mehr Anhedonie/Asozialität (für Details siehe [2]). Interessanterweise war dies auf EinzelItem-Ebene vor allem auf eine schon frühe Verschlechterung der Beziehungen mit Freunden und Peers zurückzuführen (Tab. 4).

- Tab. 3 und Tab. 4 bitte etwa hier einfügen - 


\subsection{Neuropsychologie}

Die Patienten mit späterem Übergang in Psychose (ARMS-T) hatten auch schon bei Einschluss in die Studie im Vergleich zu den Individuen ohne spätere Psychose deutlich mehr neuropsychologische Defizite gezeigt, vor allem im Bereich Arbeitsgedächtnis (Abb. 3) (für Details siehe [2]).

- Abb. 3 bitte etwa hier einfügen -

\subsection{Integriertes Prädiktionsmodell auf Basis Psychopathologie und Neuropsychologie}

In einem logistischen Regressionsmodell auf der Basis von BPRS, SANS und kognitiven Parametern konnten 3 signifikante Prädiktoren des späteren Übergangs in eine Psychose identifiziert werden: Misstrauen, Anhedonie/Asozialität sowie eine reduzierte Leistung in einer Aufgabe zur Prüfung der selektiven Aufmerksamkeit und Reaktionsinhibition (TAP [34]) - wahrscheinlich als Indikator verminderter Reaktionsgeschwindigkeit (vgl. [2]). Unter Kombination dieser Variablen konnte bei unserer durch das Basel Screening Instrument für Psychosen vorselektierten Risikopopulation die Transition mit einer Sensitivität von 83.3\% und einer Spezifität von $79.3 \%$ vorhergesagt werden (Tab. 5) (vgl. [2]).

Auf der Basis von Analysen mithilfe von Receiver-Operating-Charakteristika zeigte sich auch ganz klar, dass ein Modell unter Zuhilfenahme sowohl der Positiv- als auch der Minus-Symptomatik und der Neuropsychologie die beste Prädiktion erzielen konnte (vgl. [2]).

- Tab. 5 bitte etwa hier einfügen -

In detaillierteren Analysen zeigten sich auch spezifischere neurokognitive Differenzierungsmöglichkeiten. So waren ARMS-T schon bei der Basisuntersuchung 
bzgl. ihres verbalen episodischen Gedächtnisses im California Verbal Learning Test, CVLT [43], stärker beeinträchtigt als ARMS-NT [44].

In einer Vergleichsstudie mit Patienten mit depressiven Störungen konnten wir darüber hinaus zeigen, dass eine neuropsychologische Untersuchung auch zu der oft schwierigen Differenzialdiagnose zwischen der Prodromalphase einer Psychose und einer depressiven Störung beitragen kann. ARMS-T-Patienten zeigten signifikant mehr Defizite im Arbeitsgedächtnis und den Exekutivfunktionen als Patienten mit einer depressiven Störung [45].

\subsection{Feinmotorik}

Risiko-Individuen zeigten auch eine schlechtere Feinmotorik als gesunde Kontrollen, vor allem bezüglich Fingerfertigkeit und Geschwindigkeit der Finger-, Hand- und Arm-Aufgaben [36]. Im Rahmen einer logistischen Regression konnten auch Feinmotorik und Neuropsychologie gemeinsam analysiert werden. Hierbei ergab sich, dass die Neuropsychologie alleine $70.7 \%$, die Feinmotorik allein $69.5 \%$, die Kombination von beidem jedoch $74.4 \%$ der Individuen korrekt klassifizierte. Somit liegt in der Kombination der beiden Methoden ein deutlicher Zugewinn für die richtige Zuordnung von Individuen zur Gruppe der Risikopersonen [36].

\subsection{Neuroimaging-Prädiktoren der Transition}

In neuerer Zeit mehren sich Hinweise darauf, dass wahrscheinlich auch das MRI und fMRI des Gehirns zur Prädiktion einer Psychose beitragen können. Wie wir in einer systematischen Übersichtsarbeit und Metaanalyse zu strukturellen und funktionellen MRI-Studien zeigten, können wahrscheinlich verschiedene strukturelle und neurochemische Auffälligkeiten im präfrontalen Cortex, dem anterioren Cingulum, dem medialen, temporalen und cerebellären Cortex auf die spätere Entwicklung einer Psychose bei Risiko-Individuen hindeuten [46].

Auch auf der Basis unserer FePsy-Risikopopulation führten wir diesbezüglich verschiedene Neuroimaging-Studien durch. Zunächst zeigten wir in VBM (voxelbased morphometry) Analysen, dass ARMS-T, also Risikopatienten mit späterer Transition in Psychose, gegenüber ARMS-NT, also Risikopatienten, die keine 
Psychose entwickelten, schon bei der Aufnahmeuntersuchung geringere graue Substanzvolumina in verschiedenen Hirnregionen aufwiesen, unter anderem in der rechten Insel, im inferioren frontalen Gyrus und im anterioren, superioren und temporalen Gyrus [37,47] (Abb. 4).

- Abb. 4 bitte etwa hier einfügen -

Dieses Ergebnis war konsistent mit früheren Ergebnissen von Pantelis et al. [16] und Job et al. [48] und wurde inzwischen von Koutsouleris et al. [49] und unter Mitarbeit unserer Gruppe in multizentrischen Untersuchungen [50] bestätigt. Auch im Vergleich zu Gesunden hatten ARMS-T schon in der Prodromalphase reduzierte Volumina der grauen Substanz in verschiedenen Hirnregionen gezeigt [51]. Im längsschnittlichen Verlauf bis zur Psychose-Entwicklung zeigten sich dann bei denjenigen Patienten, die tatsächlich eine Psychose entwickelten, weitere Reduktionen der Cortex-Volumina, nicht aber bei den ARMS-Patienten, die keine Psychose entwickelten [47].

In einer anderen Studie konnten wir auf der Basis einer Analyse der kortikalen Dickenasymmetrie Risikopatienten von gesunden Kontrollen und Ersterkrankten mit einer hohen Sensitivität und Spezifität unterscheiden [52]. Ein Vergleich zwischen ARMS-T und ARMS-NT mit dieser Methodik steht noch aus, könnte aber ebenfalls erfolgsversprechend sein.

Diese MRI-Ergebnisse sind deshalb von besonderem Interesse, weil unsere Population von ARMS-Patienten neuroleptikafrei waren, was bei anderen Studien zum Teil nicht der Fall war. Neuroleptika können möglicherweise mit hirnstrukturellen Veränderungen bei Schizophrenie interagieren [53]. Allerdings bestätigte eine voxelbasierte Metaanalyse von 198 antipsychotika-naiven Risikopatienten, 206 antipsychotika-naiven Ersterkrankten und 254 Gesunden, in die auch unsere Daten eingingen, dass die Psychoseentwicklung - unabhängig von jeder neuroleptischen Behandlung - mit Reduktionen der grauen Substanz in temporalen, cingulären, cerebellären und insulären Regionen assoziiert ist [54].

Des Weiteren konnten wir in ROI (region of interest) Analysen auch Volumenveränderungen von Hippocampus, Hypophyse und Cingulum im Prozess der beginnenden Psychose zeigen. So hatten ARMS-Patienten im Basis-MRI ein 
grösseres linkes Hippocampus-Volumen als Gesunde und als Ersterkrankte mit Psychosen; auch hatten ARMS-T-Patienten ein grösseres Volumen als Ersterkrankte [55]. Dies ist konsistent mit Ergebnissen von Velakoulis et al. [56] und von Meisenzahl et al. [57]. In einer Längsschnittstudie konnten wir eine signifikante Volumenabnahme des Hippocampus bei ARMS Patienten zeigen, welche unabhängig davon war, ob die Patienten später eine Psychose entwickelten oder nicht [58].

Auch das Hypophysen-Volumen war bei ARMS-T und Ersterkrankten grösser als bei ARMS-NT, deren Volumen wiederum grösser war als bei gesunden Kontrollen. Diese Befunde könnten auf eine stressbedingte Aktivierung der hypophysären

Stresshormone ACTH und Prolaktin hindeuten, wie dies auch schon bei anderen Erkrankungen mit psychischem Stress gefunden werden konnte [59].

Schliesslich zeigten ARMS-T im Vergleich zu ARMS-NT schon im Basis-MRI signifikante Volumen-Reduktionen im Cingulum [60].

Kürzlich haben wir unsere Daten auch mit einer neuen Methode, dem sogenannten multivariaten maschinellen Lernverfahren analysiert. Auf der Basis dieser Methode konnte innerhalb der Risikopopulation mit einer Genauigkeit von 84\% die spätere Konversion vorhergesagt werden [61].

Bezüglich neurofunktioneller Parameter liessen sich in multimodalen Analysen von strukturellen und funktionellen MRI-Daten Assoziationen zur Dauer des Risikostatus zeigen [62].

\subsection{Labor}

Laboruntersuchungen führten wir unter anderem zu Prolaktin durch, da es zahlreiche Hinweise auf eine Hyperprolaktinämie und eine Störung der hypophysär-gonadalen Achse bei schizophrenen Psychosen gibt [63-65]. Insbesondere wurde immer wieder eine Hyperprolaktinämie bei diesen Patienten beobachtet, zum Teil auch schon vor und unabhängig von neuroleptischer Medikation [66]. Nicht zuletzt deuteten auch unsere Befunde zu einem vergrösserten Hypophysenvolumen im Übergang zu Psychose auf eine möglicherweise verstärkte Produktion des Stresshormons Prolaktin in der Hypophyse hin. Wir haben deshalb Prolaktinwerte zwischen unseren verschiedenen Patientengruppen verglichen und fanden bei $26 \%$ unserer neuroleptikafreien ARMS-Patienten eine Hyperprolaktinämie, und zwar vor allem bei 
Frauen [39]. Dies korrespondiert interessanterweise mit den Volumina der Hypophyse, die ja ebenfalls vor allem bei den weiblichen ARMS-T vergrössert waren. Ein Vergleich zwischen ARMS-NT und ARMS-T könnte also evtl. ebenfalls zur Prädiktion der Transition beitragen, da davon auszugehen ist, dass Patienten mit späterer psychotischer Dekompensation einen höheren Stresslevel und damit auch eine höhere Prolaktinausschüttung haben als solche ohne späteren Übergang.

\subsection{EEG}

Auch das EEG - sowohl das klinische als auch das quantitative - könnte möglicherweise eine hilfreiche Untersuchungsebene in der Prädiktion von Psychosen darstellen. So konnten wir in einer Analyse klinischer EEGs zeigen, dass Patienten mit psychotischen Ersterkrankungen und auch Risikopatienten deutlich mehr pathologische Aktivität im EEG zeigten als gesunde Kontrollprobanden. Die Zahl der Pathologien lag im Bereich früherer Studien zur Schizophrenie (z.B. Sponheim et al. [67]). Es zeigten sich aber keine spezifischen EEG-Pathologien; auch war die Pathologie unabhängig vom Einfluss psychotroper Medikamente oder von Cannabis. Die Hinzunahme der EEG-Befunde zusätzlich zur Psychopathologie (gemessen mit SANS und BPRS) erhöhte bei unveränderter Sensitivität die Spezifität der Vorhersage von 59\% auf 73\%. Das heisst, dass Individuen, die allein aufgrund ihrer Psychopathologie fälschlicherweise als Risikopatienten klassifiziert wurden, durch Mitberücksichtigung des EEGs besser als ,ohne Risiko“ klassifiziert werden konnten, wenn ihr EEG bei der Basisuntersuchung normal war $[17,68]$. Schliesslich konnten wir in Kooperation mit der Abteilung für klinische Neurophysiologie der Neurologischen Universitätsklinik Basel zusätzliche quantitative EEG-Analysen durchführen. Zunächst zeigten wir Hinweise, dass bei neuroleptika-naiven Ersterkrankten mit Psychose gewisse EEG-Frequenzbereiche mit Minussymptomatik korrelieren. Im Folgenden haben wir untersucht, ob diese Befunde auch die Prädiktion von Psychosen bei Prodromalpatienten verbessern könnten. Für sich genommen konnte weder die Minussymptomatik noch die Information aus den untersuchten EEG-Bändern die Entwicklung einer Psychose vorhersagen. Jedoch konnte die Kombination aus beiden Informationen eine signifikante Vorhersage treffen. Die beste Prädiktion mit einer korrekten Klassifikation von 89\% gelang mit einem Modell, das Minussymptomatik mit der Power im Thetafrequenzbereich kombinierte [18]. 


\section{DISKUSSION UND SCHLUSSFOLGERUNGEN}

Mit unseren verschiedenen Studien zur Früherkennung von Psychosen haben wir die eingangs aufgestellten Hypothesen weitgehend bestätigen können. Es ist tatsächlich möglich, Risiko-Individuen relativ zuverlässig mit einem gestuften Vorgehen zu identifizieren. Dieses stufenweise Vorgehen sollte am Anfang eine Risiko-Checkliste für potentielle Zuweiser sowie evtl. auch einen Selbstbeurteilungsbogen für potentiell Betroffene beinhalten. Hilfesuchende können dann mit dem von uns entwickelten Basel Screening Instrument durch speziell trainierte PsychiaterInnen untersucht werden. Aufgrund dieser einfachen Massnahme ist eine Psychose schon mit einer vergleichsweise hohen Zuverlässigkeit vorherzusagen. Die so identifizierten Individuen können vorsichtig über ein potentielles Risiko aufgeklärt werden und für die weitere Abklärung an ein spezialisiertes Früherkennungszentrum zugewiesen werden. In einem solchen Zentrum können dann weitere spezialisierte Untersuchungen durchgeführt werden, wobei es hier um drei Ziele geht:

1. Früherkennung einer schon bestehenden Psychose

2. Differentialdiagnose

3. Frühe, zuverlässige Erkennung der Prodromalphase bzw. Einschätzung des Psychose-Risikos

\section{Früherkennung einer schon bestehenden Psychose}

Wie wichtig gerade das erste Ziel eines Früherkennungszentrums ist, zeigten die ersten vier Jahre unserer Spezialsprechstunde. In dieser Zeit wurden uns 234 Individuen zugewiesen, von denen 91 schon eine klar diagnostizierbare Psychose hatten, d.h. die Dekompensationskriterien nach Yung et al. [5] erfültten. Die meisten dieser Patienten waren bisher noch nicht diagnostiziert und/oder behandelt worden. Die Dauer der unbehandelten Psychose, DUP, war in den ersten Jahren unseres FePsy-Projektes im Mittel 45 Monate (Median 12 Monate) [69]. Durch ein Früherkennungszentrum und die Bemühungen um die Zuweiser kann diese Verzögerung vermutlich massgeblich verringert und damit die deletären Konsequenzen einer lange unbehandelten Psychose vermieden werden (Übersicht bei $[1,3])$. 


\section{Differentialdiagnose}

Ein weiteres wichtiges Ziel der möglichst frühzeitigen Untersuchung in einem fraglichen Prodromalzustand ist es, andere, auch hirnorganische, Erkrankungen auszuschliessen. Bei 38 der 234 gescreenten Individuen wurden andere Diagnosen gestellt, meist ebenfalls aus dem psychiatrischen Spektrum. In mehreren Fällen steckten aber schwerwiegende organische Diagnosen hinter der Symptomatik, u.a. ein chronisch subdurales Hämatom [70], eine Enzephalitis, mehrere epileptische Erkrankungen oder eine schwere, wahrscheinlich toxisch bedingte Hirnatrophie. Diese Patienten konnten unverzüglich in weitere neurologische Behandlung überwiesen werden.

Auch fanden wir vermehrt kleinere radiologische Auffälligkeiten des Gehirns Normvarianten, aber auch pathologische Befunde. Signifikant mehr als bei Kontrollen zeigten die Ersterkrankten und die Risiko-Individuen hyper- oder hypointense Läsionen, Atrophien, hyperintense kortikale Zonen, Kavernome oder kleine lakunäre Veränderungen [71]. Gehäuft waren aber auch Auffälligkeiten wie Cavum septum pellucidum, neuroepitheliale Zysten und Virchow-Robin-Räume als mögliche Hinweise auf Hirnentwicklungsstörungen [72].

\section{Zuverlässige Erkennung der Prodromalphase bzw. Einschätzung des Psychoserisikos}

Nicht zuletzt können in einem Früherkennungszentrum weitere Abklärungen unter Einbezug verschiedener anderer Untersuchungsebenen erfolgen. Insbesondere hoffen wir, dass auf der Basis unserer Ergebnisse in Zukunft das Psychoserisiko noch exakter eingeschätzt werden kann als lediglich auf Basis des Basel Screening Instruments. So werden wir in Zukunft das Psychoserisiko als höher einschätzen, wenn fraglich prodromale Patienten starkes Misstrauen zeigen oder sich stark von ihren Freunden und Bezugspersonen zurückgezogen haben oder wenn in ihrer neuropsychologischen Testung bestimmte Auffälligkeiten vorliegen. Auch das klinische EEG kann zur genaueren Risikoeinschätzung herangezogen werden. Unter Umständen könnten auch bestimmte Laborveränderungen wie Hyperprolaktinämie zur Feststellung eines Risikostatus beitragen. Schliesslich ist absehbar, dass mit 
Hilfe neuer Analyseverfahren auf der Basis des MRI eine weitere Verbesserung der Prognosegenauigkeit möglich sein wird.

Derzeit geht es nun darum, auf der Basis dieser verschiedenen Einzelbeiträge

a) einen zeitlich gestaffelten Untersuchungsalgorithmus zu entwickeln und

b) einen individuellen Prognose-Index, der diese verschiedenen Untersuchungsebenen miteinbezieht.

Auf der Basis eines solchen Prognose-Index könnte in Zukunft ein klinisches ,Staging' bei Risiko-Individuen erfolgen [9,19,73], ähnlich wie das auch zum Teil bei somatischen Erkrankungen geschieht. Das heisst, das Risiko könnte als „gering“, „mittel“ oder „gross“ eingeschätzt werden, und die Betroffenen könnten entsprechend aufgeklärt und weiterbetreut werden. So könnte etwa bei niedrigem Risiko nur eine gute, sensible Psychoedukation und Beratung erfolgen, bei mittlerem Risiko zusätzlich eine spezifische Psychotherapie und bei Hochrisiko-Individuen evtl. zusätzlich auch eine niedrig dosierte Neuroleptika-Gabe. Andere Frühinterventionen wie etwa mit Omega-3-Fettsäuren sind derzeit noch im Forschungsstadium [3].

Zu hoffen wäre, dass Patienten mit unklaren fraglichen Prodromalzuständen künftig eine schnellere Abklärung und gezielte Frühintervention erhalten und dass damit der Ausbruch einer Psychose oder zumindest aber die schweren chronifizierenden Verläufe vermieden werden können.

Neben dieser unmittelbaren klinischen Hoffnung verbindet sich mit der Früherkennungsforschung aber auch das Ziel, mehr über die pathogenetischen Mechanismen bei der Entstehung der Erkrankung zu erfahren. 


\section{Danksagung}

Unser ganz besonderer Dank gilt allen Patienten, Angehörigen, Zuweisern, Kooperationspartnern und anderen Mitarbeitern unseres FePsy-Projektes.

Für ihre Hilfe bei der Erstellung dieses Manuskripts danken wir Frau Martina Klemm und Frau Claudine Pfister.

Das Projekt wurde finanziell unterstützt durch den Schweizerischen Nationalfonds (Nr. 3200057216.99, 3200-0572216.99, PBBSB-106936, und 3232BO-119382); die Nora van Meeuwen-Haefliger Stiftung, Basel $(\mathrm{CH})$ und - ohne Verpflichtungen - durch die Novartis Foundation; Bristol-Myers Squibb, GmbH (CH); Eli Lilly SA (CH); AstraZeneca AG (CH); Janssen-Cilag $A G(C H)$, und Sanofi-Synthelabo $A G(C H)$. 


\section{Legenden für Tabellen und Abbildungen}

Tabelle 1a: Risiko für Psychosen - eingeschätzt mit dem „Basel Screening Instrument für Psychosen“ (BSIP) [22]

Tabelle 1b: Dekompensationskriterien der Psychose nach Yung et al. [5]

Tabelle 2: Übergänge in Psychosen und Beobachtungsdauer bei nicht dekompensierten ARMS-Individuen im Basler FePsy-Projekt

Tabelle 3: $\quad$ Psychopathologie (BPRS) bei Studienbeginn - Risikoindividuen ohne (ARMS-NT) versus mit späterer Transition in Psychose (ARMS-T)

Tabelle 4: $\quad$ Anhedonie/Asozialität (SANS) bei Studienbeginn - Risikoindividuen ohne (ARMS-NT) versus mit späterer Transition in Psychosen (ARMS-T)

Tabelle 5: Integriertes Modell zur Prädiktion der Transition basierend auf BPRSund SANS-Subskalen Scores und Neuropsychologie

Abbildung 1: Risikocheckliste für Psychosen ${ }^{\circledR}$ Riecher-Rössler

Abbildung 2: Selbstbeurteilungsfragebogen zur Früherkennung von Psychosen [23]

Abbildung 3: Neuropsychologische Profile bei Studienbeginn - ARMS-T (mit späterer Transition) im Vergleich zu ARMS-NT (ohne spätere Transition in Psychose)

Abbildung 4: Areale reduzierter grauer Hirnsubstanz-Volumina bei ARMSIndividuen mit späterer Transition in Psychose (ARMS-T) verglichen mit ARMS-Individuen ohne Entwicklung einer Psychose (ARMS-NT) 


\section{LITERATUR}

1. Riecher-Rössler A, Gschwandtner U, Borgwardt S et al. Early detection and treatment of schizophrenia: how early? Acta Psychiatr Scand Suppl 2006: 73-80

2. Riecher-Rössler A, Pflueger MO, Aston $\mathrm{J}$ et al. Efficacy of using cognitive status in predicting psychosis: a 7-year follow-up. Biol Psychiatry 2009; 66: 1023-1030

3. McGorry PD, Nelson B, Amminger GP et al. Intervention in individuals at ultra high risk for psychosis: a review and future directions. J Clin Psychiatry 2009; 70: 1206-1212

4. Fusar-Poli P, Borgwardt S, Bechdolf A et al. The Psychosis High-Risk State: A Comprehensive State-of-the-Art Review. JAMA Psychiatry 2013; 70: 107-120

5. Yung AR, Phillips LJ, McGorry PD et al. Prediction of psychosis. A step towards indicated prevention of schizophrenia. Br J Psychiatry Suppl 1998; 172: 14-20

6. Yung AR, Woods SW, Ruhrmann S et al. Editorial: whither the attenuated psychosis syndrome? Schizophr Bull 2012; accepted

7. Yung AR, Yuen HP, Berger $G$ et al. Declining transition rate in ultra high risk (prodromal) services: dilution or reduction of risk? Schizophr Bull 2007; 33: 673-681

8. Cannon TD, Cadenhead K, Cornblatt B et al. Prediction of psychosis in youth at high clinical risk. Arch Gen Psychiatry 2008; 65: 28-37

9. Ruhrmann S, Schultze-Lutter F, Salokangas RK et al. Prediction of psychosis in adolescents and young adults at high risk: results from the prospective European prediction of psychosis study. Arch Gen Psychiatry 2010; 67: 241-251

10. Fusar-Poli P, Bonoldi I, Yung AR et al. Predicting psychosis: meta-analysis of transition outcomes in individuals at high clinical risk. Arch Gen Psychiatry 2012; 69: 220-229

11. Klosterkötter J, Hellmich M, Steinmeyer EM et al. Diagnosing schizophrenia in the initial prodromal phase. Arch Gen Psychiatry 2001; 58: 158-164

12. Koutsouleris N, Davatzikos C, Bottlender R et al. Early recognition and disease prediction in the at-risk mental states for psychosis using neurocognitive pattern classification. Schizophr Bull 2011; Epub Date 2011/05/18: DOI 10.1093/schbul/sbr1037

13. Seidman LJ, Giuliano AJ, Meyer EC et al. Neuropsychology of the prodrome to psychosis in the NAPLS consortium: relationship to family history and conversion to psychosis. Arch Gen Psychiatry 2010; 67: 578-588 
14. Koutsouleris N, Meisenzahl EM, Davatzikos C et al. Use of neuroanatomical pattern classification to identify subjects in at-risk mental states of psychosis and predict disease transition. Arch Gen Psychiatry 2009; 66: 700-712

15. Koutsouleris N, Gaser C, Bottlender R et al. Use of neuroanatomical pattern regression to predict the structural brain dynamics of vulnerability and transition to psychosis. Schizophr Res 2010; 123: 175-187

16. Pantelis C, Velakoulis D, McGorry PD et al. Neuroanatomical abnormalities before and after onset of psychosis: a cross-sectional and longitudinal MRI comparison. Lancet 2003; 361: 281 288

17. Gschwandtner U, Pflueger MO, Semenin V et al. EEG: a helpful tool in the prediction of psychosis. Eur Arch Psychiatry Clin Neurosci 2009; 259: 257-262

18. Zimmermann R, Gschwandtner U, Wilhelm FH et al. EEG spectral power and negative symptoms in at-risk individuals predict transition to psychosis. Schizophr Res 2010; 123: 208216

19. Bodatsch M, Ruhrmann S, Wagner M et al. Prediction of psychosis by mismatch negativity. Biological Psychiatry 2011; 69: 959-966

20. van Tricht MJ, Nieman DH, Koelman JHTM et al. Reduced parietal P300 amplitude is associated with an increased risk for a first psychotic episode. Biological Psychiatry 2010; 68: 642-648

21. Riecher-Rössler A, Gschwandtner U, Aston J et al. The Basel early-detection-of-psychosis (FEPSY)-study - design and preliminary results. Acta Psychiatr Scand 2007; 115: 114-125

22. Riecher-Rössler A, Aston J, Ventura J et al. Das Basel Screening Instrument fur Psychosen (BSIP): Entwicklung, Aufbau, Reliabilität und Validität. Fortschr Neurol Psychiatr 2008; 76: 207216

23. Kammermann J, Stieglitz RD, Riecher-Rössler A. "Selbstscreen-Prodrom" - Ein Selbstbeurteilungsinstrument zur Früherkennung von psychischen Erkrankungen und Psychosen. Fortschr Neurol Psychiatr 2009; 77: 278-284

24. American Psychiatric Association. Diagnostic and Statistical Manual of Mental Disorders, 3rd ed. rev. Washington, DC: American Psychiatric Press; 1987

25. Lukoff $\mathrm{D}$, Nuechterlein $\mathrm{KH}$, Ventura J. Manual for the expanded brief psychiatric rating scale. Schizophr Bull 1986; 12: 594-602 
26. Ventura J, Lukoff $\mathrm{D}$, Nuechterlein $\mathrm{KH}$ et al. Training and quality assurance with the brief psychiatric rating scale: "The Drift Busters"; Appendix 1 the Brief Psychiatric Rating Scale (expanded Version). Int J Methods Psychiatr Res 1993; 3: 221-224

27. Yung AR, McGorry PD, Francey SM et al. PACE: a specialised service for young people at risk of psychotic disorders. Med J Aust 2007; 187: S43-46

28. Andreasen NC. The scale for the assessment of negative symptoms (SANS): conceptual and theoretical foundations. Br J Psychiatry Suppl 1989: 49-58

29. Lehrl S. Manual zum MWT. Balingen, Germany: Perimed; 1991

30. Horn W. Leistungsprüfsystem (LPS), 2nd ed. Göttingen, Toronto, Zürich: Verlag für Psychologie; 1983

31. Gedika G, Schöttke H. Der Turm von Hanoi - TvH. Hogrefe Testsystem (HTS). Göttingen: Hogrefe; 1994

32. Drühe-Wienholt C, Wienholt W. CKV: Computergestütztes Kartensortierverfahren. Frankfurt am Main: Swets und Zeitlinger Testservices; 1998

33. Heaton RK, Chelune GH, Talley JL et al. Wisconsin Card Sorting Test Manual. Odessa, Florida: Psychological Assessment Resources; 1993

34. Zimmermann P, B. F. Testbatterie zur Aufmerksamkeitsprüfung (TAP), Version 1.20: Handbuch. Würselen: Vera Fimm/Psychologische Testsysteme; 1993

35. Rosvold HE, Mirsky AF, Sarason I et al. A continuous performance test of brain damage. J Cons Psychol 1965; 20: 343-350

36. Gschwandtner U, Pflüger M, Aston J et al. Fine motor function and neuropsychological deficits in individuals at risk for schizophrenia. Eur Arch Psychiatry Clin Neurosci 2006; 256: 201-206

37. Borgwardt SJ, Riecher-Rössler A, Dazzan $P$ et al. Regional gray matter volume abnormalities in the at risk mental state. Biol Psychiatry 2007; 61: 1148-1156

38. Smieskova R, Fusar-Poli P, Aston J et al. Insular volume abnormalities associated with different transition probabilities to psychosis. Psychological Medicine 2012; 42: 1613-1625

39. Aston J, Rechsteiner E, Bull $\mathrm{N}$ et al. Hyperprolactinaemia in early psychosis - not only due to antipsychotics. Prog Neuropsychopharmacol Biol Psychiatry 2010; 34: 1342-1344

40. Pukrop R, Ruhrmann S, Schultze-Lutter F et al. Neurocognitive indicators for a conversion to psychosis: comparison of patients in a potentially initial prodromal state who did or did not convert to a psychosis. Schizophr Res 2007; 92: 116-125 
41. Salthouse TA, Hedden T. Interpreting reaction time measures in between-group comparisons. J Clin Exp Neuropsychol 2002; 24: 858-872

42. DeLong ER, DeLong DM, Clarke-Pearson DL. Comparing the areas under two or more correlated receiver operating characteristic curves: a nonparametric approach. Biometrics 1988; 44: 837-845

43. Delis DC, Kramer JH, Kaplan E et al. California verbal learning test, second edition (CVLT-II): Pearson; 2000

44. Zimmermann R, Gschwandtner U, Pflueger $M$ et al. Verbal episodic memory deficits in patients with first episode psychosis and individuals with an at risk mental state. 2011; in Revision

45. Schulze $\mathrm{C}$, Zimmermann $\mathrm{R}$, Gschwandtner $\mathrm{U}$ et al. Can cognitive deficits facilitate differential diagnosis between at-risk mental state for psychosis and depressive disorders? Early Interv Psychiatry 2012;

46. Smieskova R, Fusar-Poli $\mathrm{P}$, Allen $\mathrm{P}$ et al. Neuroimaging predictors of transition to psychosis--a systematic review and meta-analysis. Neurosci Biobehav Rev 2010; 34: 1207-1222

47. Borgwardt SJ, Fusar-Poli $\mathrm{P}$, Radü EW et al. Insular pathology in the at-risk mental state. Eur Arch Psychiatry Clin Neurosci 2008; 258: 254-255

48. Job DE, Whalley HC, Johnstone EC et al. Grey matter changes over time in high risk subjects developing schizophrenia. Neuroimage 2005; 25: 1023-1030

49. Koutsouleris N, Schmitt GJ, Gaser C et al. Neuroanatomical correlates of different vulnerability states for psychosis and their clinical outcomes. Br J Psychiatry 2009; 195: 218-226

50. Mechelli A, Riecher-Rössler A, Meisenzahl EM et al. Neuroanatomical abnormalities that predate the onset of psychosis: a multicenter study. Arch Gen Psychiatry 2011; 68: 489-495

51. Borgwardt SJ, McGuire PK, Aston J et al. Structural brain abnormalities in individuals with an atrisk mental state who later develop psychosis. Br J Psychiatry Suppl 2007; 51: 69-75

52. Haller S, Borgwardt SJ, Schindler $\mathrm{C}$ et al. Can cortical thickness asymmetry analysis contribute to detection of at-risk mental state and first-episode psychosis? A pilot study. Radiology 2009; 250: $212-221$

53. Borgwardt SJ, Riecher-Rössler A, Smieskova R et al. Superior temporal gray and white matter changes in schizophrenia or antipsychotic related effects? Schizophr Res 2009; 113: 109-110

54. Fusar-Poli P, Radua J, McGuire $P$ et al. Neuroanatomical maps of psychosis onset: voxelwise meta-analysis of antipsychotic-naive VBM studies. Schizophr Bull 2011; im Druck 
55. Buehlmann E, Berger GE, Aston J et al. Hippocampus abnormalities in at risk mental states for psychosis? A cross-sectional high resolution region of interest magnetic resonance imaging study. J Psychiatr Res 2010; 44: 447-453

56. Velakoulis D, Wood SJ, Wong MT et al. Hippocampal and amygdala volumes according to psychosis stage and diagnosis: a magnetic resonance imaging study of chronic schizophrenia, first-episode psychosis, and ultra-high-risk individuals. Arch Gen Psychiatry 2006; 63: 139-149

57. Meisenzahl EM, Koutsouleris N, Gaser C et al. Structural brain alterations in subjects at highrisk of psychosis: a voxel-based morphometric study. Schizophr Res 2008; 102: 150-162

58. Walter A, Studerus E, Smieskova $R$ et al. Hippocampal volume in subjects at high risk of psychosis: A longitudinal MRI study. Schizophr Res 2012; 142: 217-222

59. Büschlen J, Berger GE, Borgwardt SJ et al. Pituitary volume increase during emerging psychosis. Schizophr Res 2011; 125: 41-48

60. Rothlisberger $\mathrm{M}$, Riecher-Rossler $\mathrm{A}$, Aston $\mathrm{J}$ et al. Cingulate volume abnormalities in emerging psychosis. Curr Pharm Design 2012; 18: 495-504

61. Koutsouleris N, Borgwardt S, Meisenzahl EM et al. Disease prediction in the at-risk mental state for psychosis using neuroanatomical biomarkers: results from the FePsy study. Schizophrenia Bulletin 2011;

62. Smieskova $\mathrm{R}$, Allen $\mathrm{P}$, Simon $\mathrm{A}$ et al. Different duration of at-risk mental state associated with neurofunctional abnormalities. A multimodal imaging study. Hum Brain Mapp 2011; Epub Date 2011/09/17: DOI 10.1002/hbm.21360

63. Riecher-Rössler A, Häfner H. Schizophrenia and oestrogens - is there an association? Eur Arch Psychiatry Clin Neurosci 1993; 242: 323-328

64. Riecher-Rössler A, Häfner H, Dütsch-Strobel A et al. Gonadal function and its influence on psychopathology. Arch Womens Ment Health 1998; 1: 15-26

65. Riecher-Rössler A, Schmid C, Bleuer S et al. Antipsychotika und Hyperprolaktinämie: Pathophysiologie, klinische Bedeutung, Abklärung und Therapie. Neuropsychiatr 2009; 23: 71 83

66. Kahn RS, Fleischhacker WW, Boter $\mathrm{H}$ et al. Effectiveness of antipsychotic drugs in first-episode schizophrenia and schizophreniform disorder: an open randomised clinical trial. Lancet 2008; 371: 1085-1097

67. Sponheim SR, Clementz BA, lacono WG et al. Resting EEG in first-episode and chronic schizophrenia. Psychophysiology 1994; 31: 37-43 
68. Pflüger M, Gschwandtner U, Kälin A et al. Was kann das EEG zur Vorhersage von schizophrenen Psychosen leisten? neuro aktuell - Informationsdienst für Neurologen und Psychiater 2010; 01: 30-35

69. Fridgen G. Hilfesuchverhalten bei beginnender Psychose. Vergleich zwischen Risikopersonen für eine Psychose und Ersterkrankten sowie zwischen Männern und Frauen. Dissertation: Medizinische Fakultät. Universität Basel; 2011:82

70. Gschwandtner U, Borgwardt S, Aston J et al. Chronisch subdurales Hämatom bei einem Patienten mit Verdacht auf Prodromalstadium einer Schizophrenie. Nervenarzt 2004; 75: 691693

71. Borgwardt SJ, Radue EW, Götz K et al. Radiological findings in individuals at high risk of psychosis. J Neurol Neurosurg Psychiatry 2006; 77: 229-233

72. Borgwardt SJ, Radü EW, Riecher-Rössler A. Cavum septum pellucidum in patients with first episode psychosis and individuals at high risk of psychosis. Eur Psychiatry 2007; 22: 264

73. Agius $\mathrm{M}$, Goh $\mathrm{C}$, Ulhaq S et al. The staging model in schizophrenia, and its clinical implications. Psychiatr Danub 2010; 22: 211-220 
Tabelle 1a: Risiko für Psychosen - eingeschätzt mit dem „Basel Screening Instrument für Psychosen“ (BSIP) [22]

I Das Individuum hat ein Risiko, eine schizophrene Psychose zu entwickeln, wenn es eines der folgenden Kriterien erfüllt:

State [5]

- $\quad$ "Attenuierte" psychotische Symptome:

Psychotische Symptome unter Dekompensationsschwelle

(Skalen: Halluzinationen 2-3, ungewöhnliche Denkinhalte 3-4,

Misstrauen 3-4, mindestens mehrmals pro Woche, insgesamt $>1$ Woche anhaltend

ODER

- $\quad$ BLIPS - Brief Limited Intermittent Psychotic Symptoms:

Psychotische Symptome über Dekompensationsschwelle

(Halluzinationen $\geq 4$, ungewöhnliche Denkinhalte $\geq 5$, Misstrauen $\geq 5$,

Denkzerfahrenheit $\geq 5$ )

jedes Symptom kürzer als 1 Woche mit spontaner Remission

II Trait + State

Erst- oder zweitgradig Verwandter mit Psychose und weitere Risikofaktoren laut Screeningbogen (BSIP)

III Mischkategorie

Kombination verschiedener Risikofaktoren laut Screeningbogen (BSIP)

Aber Dekompensationskriterien für Psychose noch nicht erfüllt (s. Tab. 1b)! 
Tabelle 1b: Dekompensationskriterien der Psychose nach Yung et al. [5]

- Mindestens eines der folgenden Symptome:

Misstrauen (BPRS ${ }^{1} \geq 5$ )

Sagt, dass andere böswillig über ihn reden, böse Absichten haben oder ihn schädigen wollen (Vorfälle >1/Woche ODER teilweise wahnhafte Überzeugung)

Ungewöhnliche Denkinhalte (BPRS $\geq 5$ )

Eindeutiger Wahn mit Überzeugung ODER einigen gestörten

Funktionsbereichen (nicht nur Beziehungsideen, Verfolgungsideen, ungewöhnlicher Glaube oder bizarre Vorstellungen ohne feste wahnhafte Überzeugungen)

Halluzinationen (BPRS $\geq 4$ )

Gelegentliche Halluzinationen ODER visuelle Halluzinationen $>2$ /Woche oder mit funktioneller Einschränkung (nicht nur Hören des eigenen Namens, non-verbale akustische oder formlose visuelle Halluzinationen)

\section{Formale Denkstörungen (BPRS $\geq 5$ )}

Sprache wegen Umständlichkeit, Sprunghaftigkeit, Neologismen, Sperrungen oder Themenwechsel schwer verständlich (meistens ODER 3-5 Beispiele für inkohärente Redewendungen)

- Symptome wenigstens mehrmals pro Woche

- Veränderung des Psychostatus länger als eine Woche

${ }^{1}$ BPRS (Brief Psychiatric Rating Scale) nach Lukoff et al. [25] 
Tabelle 2: Übergänge in Psychosen und Beobachtungsdauer bei nicht dekompensierten ARMS-Individuen im Basler FePsy-Projekt ${ }^{1}$

\begin{tabular}{lcccc}
\hline $\begin{array}{l}\text { Zeitraum } \\
\text { (Monate) }\end{array}$ & $\begin{array}{c}\text { Beobachtungs- } \\
\text { ende bei ARMS- } \\
\text { NT }^{2}\end{array}$ & $\begin{array}{c}\text { Anzahl } \\
\text { Transitionen }\end{array}$ & $\begin{array}{c}\text { Kumulative } \\
\text { Transitionsrate }\end{array}$ & $\begin{array}{c}95 \% \\
\text { Confidence- } \\
\text { Intervall }\end{array}$ \\
\hline $0-6$ & - & 9 & .14 & $.05-.22$ \\
7.12 & - & 6 & .23 & $.12-.33$ \\
$13-18$ & - & 2 & .27 & $.15-.36$ \\
$19-24$ & - & 2 & .30 & $.18-.40$ \\
$25-30$ & - & 0 & .30 & $.11-.40$ \\
$31-36$ & - & 1 & .31 & $.19-42$ \\
$37-84$ & 32 & 1 & .34 & $.21-.46$
\end{tabular}

Kaplan-Meier-Schätzungen geschichtet für 6-Monats-Intervalle, basierend auf der Beobachtung von $\mathrm{n}=64$ Risikoindividuen über maximal 7, im Mittel 5.4 Jahre.

Quelle: [2]

${ }^{2}$ ARMS-NT: At-Risk Mental State Individuen ohne Transition in eine Psychose 
Tabelle 3: Psychopathologie (BPRS) ${ }^{1}$ bei Studienbeginn - Risikoindividuen ohne (ARMS-NT) versus mit späterer Transition in Psychose (ARMS-T)

\begin{tabular}{lccccc}
\hline & \multicolumn{2}{c}{$\begin{array}{c}\text { ARMS-NT } \\
(\mathrm{n}=32)\end{array}$} & \multicolumn{2}{c}{ ARMS-T } & Signifikanz \\
& $\mathrm{M}$ & $\mathrm{n}=21)$ & \\
\cline { 2 - 6 } & 1.3 & $(0.7)$ & 1.7 & $(1.1)$ & $\mathrm{n}$ \\
\hline Halluzinationen & 2.0 & $(1.1)$ & 2.3 & $(1.4)$ & n.s. \\
Ungewöhnliche Denkinhalte & 1.9 & $(1.0)$ & 3.0 & $(1.3)$ & $*$ ** \\
Misstrauen & 1.5 & $(0.8)$ & 1.8 & $(1.0)$ & n.s. \\
Formale Denkstörungen & 37.8 & $(8.3)$ & 42.4 & $(9.8)$ & $\circ$ \\
Global-Score & & & & \\
\hline
\end{tabular}

Einschluss 01.03 .2000 - 29.02.2004, Follow-up bis 28.02.2007

M: Mittelwert; SD: Standardabweichung

${ }^{1}$ BPRS: Brief Psychiatric Rating Scale [25]

${ }^{2}$ Mann-Whitney U-Test zweiseitig (adjustiert für multiple Testungen);

n.s.: nicht signifikant; ${ }^{\circ}: p<.1 ;{ }^{*}: p<.05 ;^{* *}: p<.01$

Quelle: [2] 
Tabelle 4: Anhedonie/Asozialität (SANS ${ }^{1}$ ) bei Studienbeginn - Risikoindividuen ohne (ARMS-NT) versus mit späterer Transition in Psychosen (ARMS-T)

\begin{tabular}{lccccccc}
\hline $\begin{array}{l}\text { Subskala Anhedonie/Asozialität } \\
\text { Einzel-Items }\end{array}$ & \multicolumn{3}{c}{$\begin{array}{c}\text { ARMS-NT } \\
(\mathrm{n}=32)\end{array}$} & \multicolumn{3}{c}{$\begin{array}{c}\text { ARMS-T } \\
(\mathrm{n}=21)\end{array}$} & $\begin{array}{c}\text { Signifikanz } \\
\mathrm{p}^{2}\end{array}$ \\
\hline & $\mathrm{n}^{3}$ & $\mathrm{M}$ & $\mathrm{SD}$ & $\mathrm{n}^{3}$ & $\mathrm{M}$ & $\mathrm{SD}$ & \\
\cline { 2 - 8 } & & & & & & & \\
& 32 & 1.6 & $(1.5)$ & 20 & 2.8 & $(1.5)$ & $*$ \\
$\begin{array}{l}\text { Freizeitinteressen und -aktivitäten } \\
\text { Sexuelles Interesse und Aktivität }\end{array}$ & 20 & 1.0 & $(1.4)$ & 7 & 2.0 & $(1.3)$ & n.s. \\
$\begin{array}{l}\text { Fähigkeit, Nähe und Intimität zu } \\
\text { empfinden }\end{array}$ & 28 & 1.1 & $(1.4)$ & 12 & 2.4 & $(1.6)$ & $\circ$ \\
$\begin{array}{l}\text { Beziehungen mit Freunden und } \\
\text { Peers }\end{array}$ & 32 & 1.3 & $(1.4)$ & 18 & 2.7 & $(1.3)$ & $*$ \\
$\begin{array}{l}\text { Globaleinschätzung von } \\
\text { Anhedonie/Asozialität }\end{array}$ & 32 & 1.4 & $(1.4)$ & 20 & 2.7 & $(1.6)$ & $* *$ \\
\hline
\end{tabular}

Einschluss 01.03.2000 - 29.02.2004, Follow-up bis 28.02.2007

${ }^{1}$ Scale for the Assessment of Negative Symptoms [28]

${ }^{2}$ Mann-Whitney U-Test zweiseitig (adjustiert für multiple Testungen);

n.s.: nicht signifikant, ${ }^{\circ}: p<.1 ;{ }^{*}: p<.05 ;{ }^{* *} p<.01$

${ }^{3} \mathrm{n}$ unterschiedlich aufgrund fehlender Daten 
Tabelle 5: Integriertes Modell ${ }^{1}$ zur Prädiktion der Transition basierend auf BPRSund SANS-Subskalen Scores und Neuropsychologie

\begin{tabular}{lcc}
\hline Ausgewählte Variablen & $\boldsymbol{\beta}$ & Odds Ratio \\
\hline Konstanter Term & $-4.90[-8.36--2.48]$ & $0.01[0.00-0.08]$ \\
BPRS Misstrauen & $1.09[0.43-1.96]$ & $2.97[1.54-7.07]$ \\
TAP-Go/NoGo (Fehler/Zeit) & $-1.04[-2.09--0.17]$ & $0.35[0.12-0.84]$ \\
SANS Anhedonie/Asozialität & $0.24[0.07-0.47]$ & $1.27[1.07-1.59]$ \\
\hline
\end{tabular}

$\mathrm{n}=18$ ARMS-T vs. $\mathrm{n}=29$ ARMS-NT

4 Iterationen resultieren in: Test von Modell-Koeffizienten

$\mathrm{Chi}^{2}=22.02(\mathrm{df}=4), \mathrm{p} \leq .0001$
-2 Log-Likelihood $=40.5$

Cox \& Snell $\mathrm{R}^{2}=.374$

Nagelkerke $R^{2}=.508$

$\mathrm{AUC}=0.87$

\section{Klassifikation}

\begin{tabular}{lccll}
\hline & \multicolumn{3}{c}{ Prädiziert } \\
\cline { 2 - 4 } Beobachtet & ARMS-NT & ARMS-T & \\
\cline { 2 - 4 } ARMS-NT & 23 & 6 & 79.3 (Spezifität) & 88.5 (negativer prädiktiver Wert) \\
ARMS-T & 3 & 15 & 83.3 (Sensitivität) & 71.4 (positiver prädiktiver Wert) \\
\cline { 2 - 4 } & \multicolumn{5}{c}{80.9 (Gesamtgenauigkeit) } \\
\hline
\end{tabular}

${ }^{1}$ Schrittweise logistische Regression mit Rückwärtseliminations-Algorithmus (Wald-Statistik) Quelle: [2] 


\title{
Abbildung 1: Risikocheckliste für Psychosen ${ }^{\circledR}$ Riecher-Rössler
}

\begin{abstract}
Wenn bei jungen Menschen anhaltende Veränderungen im Wesen oder in ihrer Leistung auftreten, sollte man immer an die Möglichkeit einer beginnenden Psychose denken. Achten Sie bitte auf folgende mögliche Frühzeichen oder Risikofaktoren, insbesondere wenn diese in den letzten Jahren neu aufgetreten sind.
\end{abstract}

\section{A Frühsymptome}

\section{Veränderungen des Wesens}

$\square$ Unruhe, Nervosität, Reizbarkeit

$\square$ Erhöhte Sensibilität, Überempfindlichkeit, Irritierbarkeit

$\square$ Schlafstörungen, Appetitlosigkeit

$\square$ Selbstvernachlässigung

$\square$ Plötzlicher Mangel an Interesse, Initiative, Energie, Antrieb

\section{Veränderungen der Gefühle}

$\square$ Depression, verflachte Gefühle oder starke Stimmungsschwankungen

$\square$ Ängste, vor allem Angst, geschädigt/bedroht zu werden

\section{Veränderungen der Leistungsfähigkeit}

$\square$ Geringe Belastbarkeit, v.a. bei Stress

$\square$ Konzentrationsstörungen, erhöhte Ablenkbarkeit

$\square$ Leistungsknick

\section{Veränderungen im sozialen Bereich}

$\square$ Misstrauen

$\square$ Sozialer Rückzug, Isolation

$\square$ Probleme in Beziehungen

\section{Veränderungen der Interessen}

$\square$ Plötzlich ungewöhnliche Interessen, z.B. für Religiöses oder übernatürliche Dinge, Magie etc.

\section{Veränderungen der Wahrnehmung und des Erlebens}

$\square$ Eigentümliche Vorstellungen, ungewöhnliches Erleben

$\square$ Ungewöhnliche Wahrnehmungen

$\square$ (z.B. Intensivierung oder Veränderung von Geräuschen oder Farben; Gefühl, man selbst oder die Umgebung sei verändert)

$\square$ Eigenbeziehungen (bezieht alles auf sich); Gefühl, beobachtet zu werden

$\square$ Beeinflussungserleben

$\square$ (Gefühl, andere können auf mich oder meine Gedanken Zugriff nehmen,

$\square$ mich kontrollieren, steuern)

$\square$ Ungewöhnliches hören oder sehen (wenn niemand/nichts anwesend ist)

\section{B „Knick“ in der Lebenslinie}

$\square$ Auffälliger Leistungsknick, Schwierigkeiten im Beruf, in der Schule etc.

$\square$ Beruflicher Abstieg, Verlust des Ausbildungs- oder Arbeitsplatzes

$\square$ Auffällige Verschlechterung in der Beziehungsfähigkeit (Partnerschaft, Familie, Beruf etc.)

\section{Psychische Erkrankungen in der Verwandtschaft}

$\square$ Psychose in der Familie/Verwandtschaft bekannt oder zu vermuten

$\square$ Psychische Erkrankungen in der Herkunftsfamilie

\section{Drogen}

$\square$ Regelmässiger Konsum von Drogen (Alkohol, Cannabis, Kokain, Opiate, Amphetamine etc.)

\section{E Alter}

$\square$ Ist die Patientin/der Patient unter 30 Jahre alt (auch späterer Beginn ist möglich)? 
Abbildung 2: Selbstbeurteilungsfragebogen zur Früherkennung von Psychosen [23]

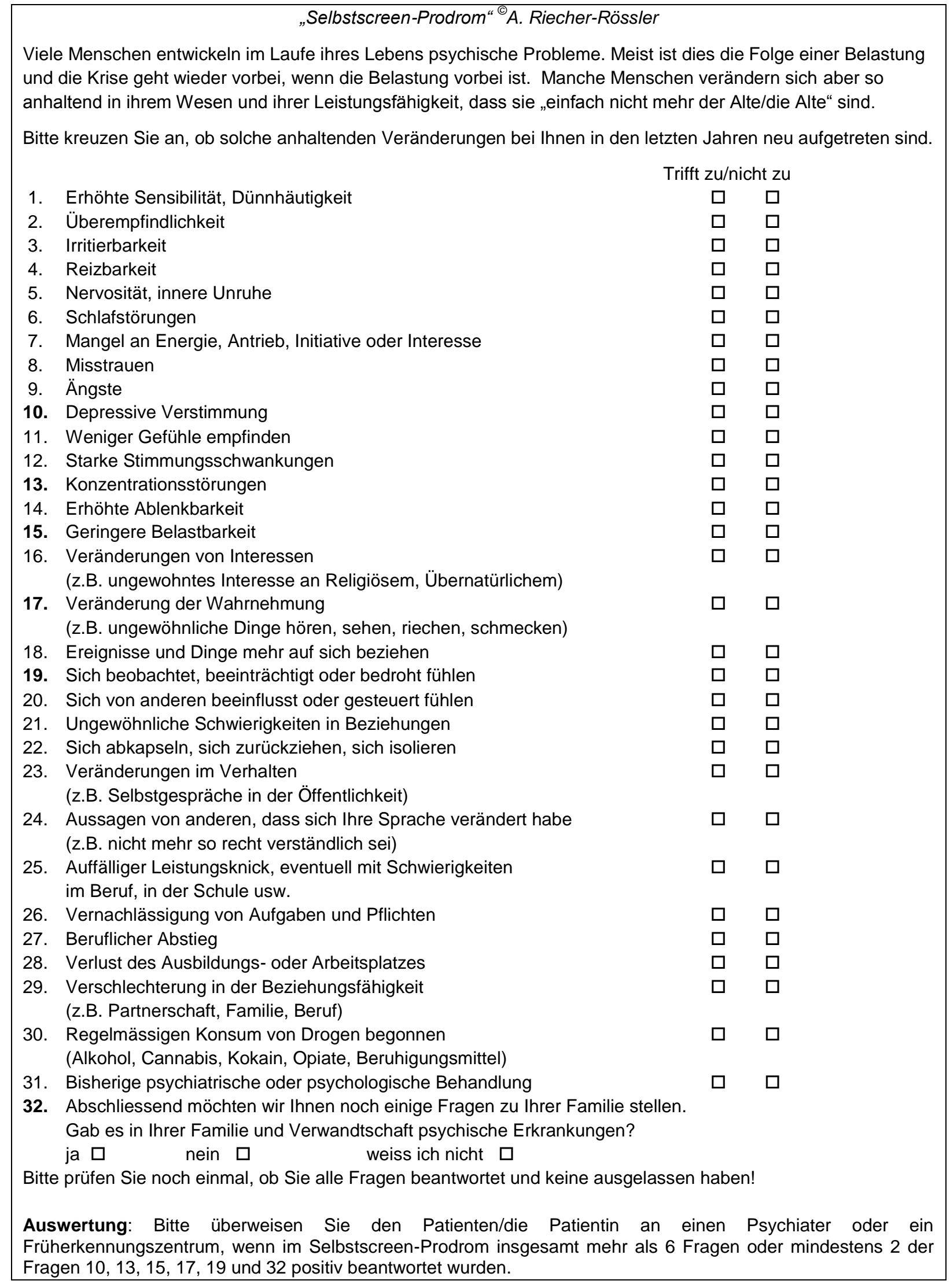


Abbildung 3: Neuropsychologische Profile bei Studienbeginn - ARMS-T (mit späterer Transition) im Vergleich zu ARMS-NT (ohne spätere Transition in Psychose)

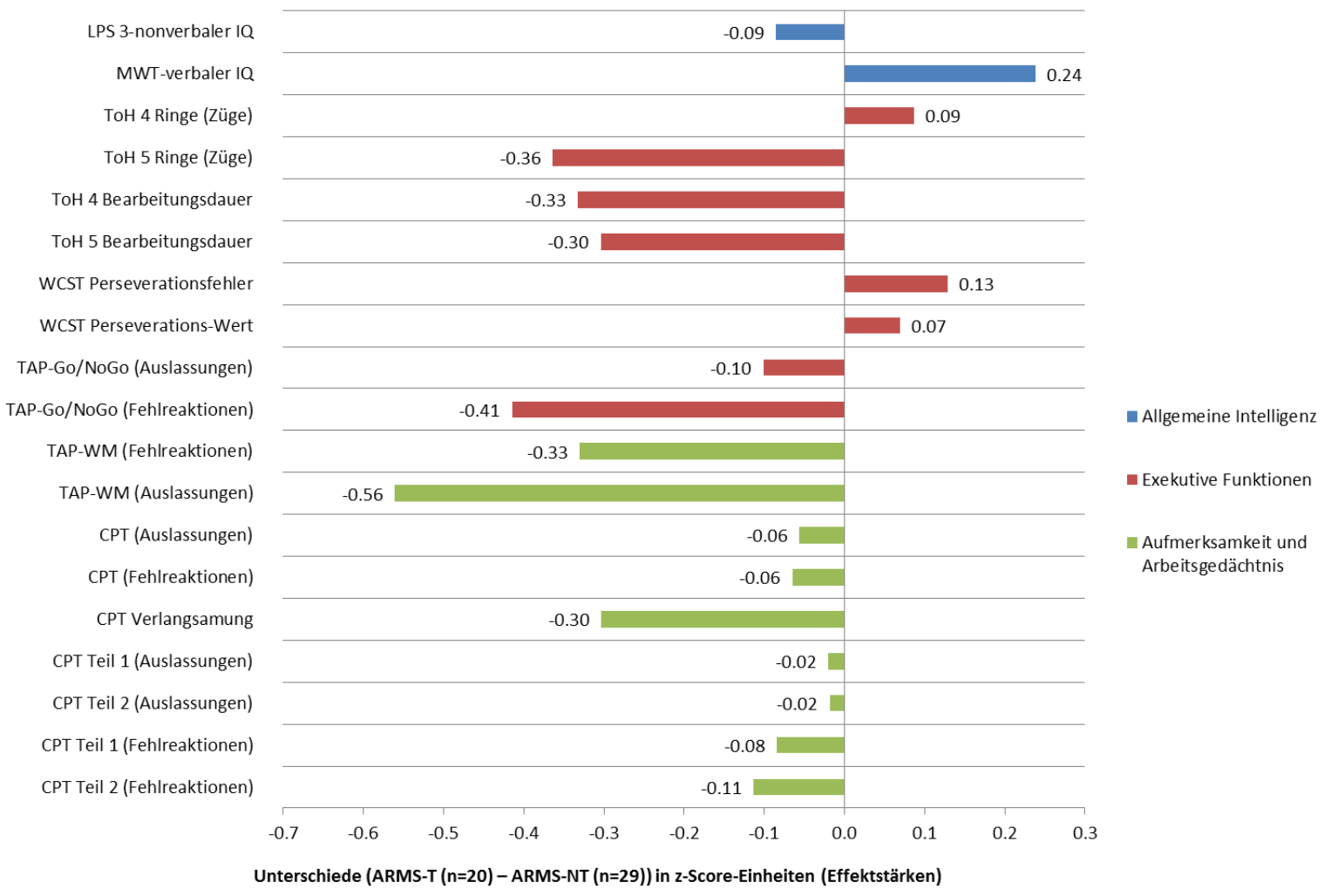

LPS 3: Leistungsprüfungssystem [30]

MWT: Mehrfachwahl-Wortschatz-Intelligenztest [29]

ToH: Tower of Hanoi [31]

WCST: Wisconsin Card Sorting Test [33]

TAP: Testbatterie zur Aufmerksamkeitsprüfung [34]

CPT: $\quad$ Continuous Performance Test [35] 
Abbildung 4: Areale reduzierter grauer Hirnsubstanz-Volumina bei ARMS-Individuen mit späterer Transition in Psychose (ARMS-T) verglichen mit ARMS-Individuen ohne Entwicklung einer Psychose (ARMS-NT)

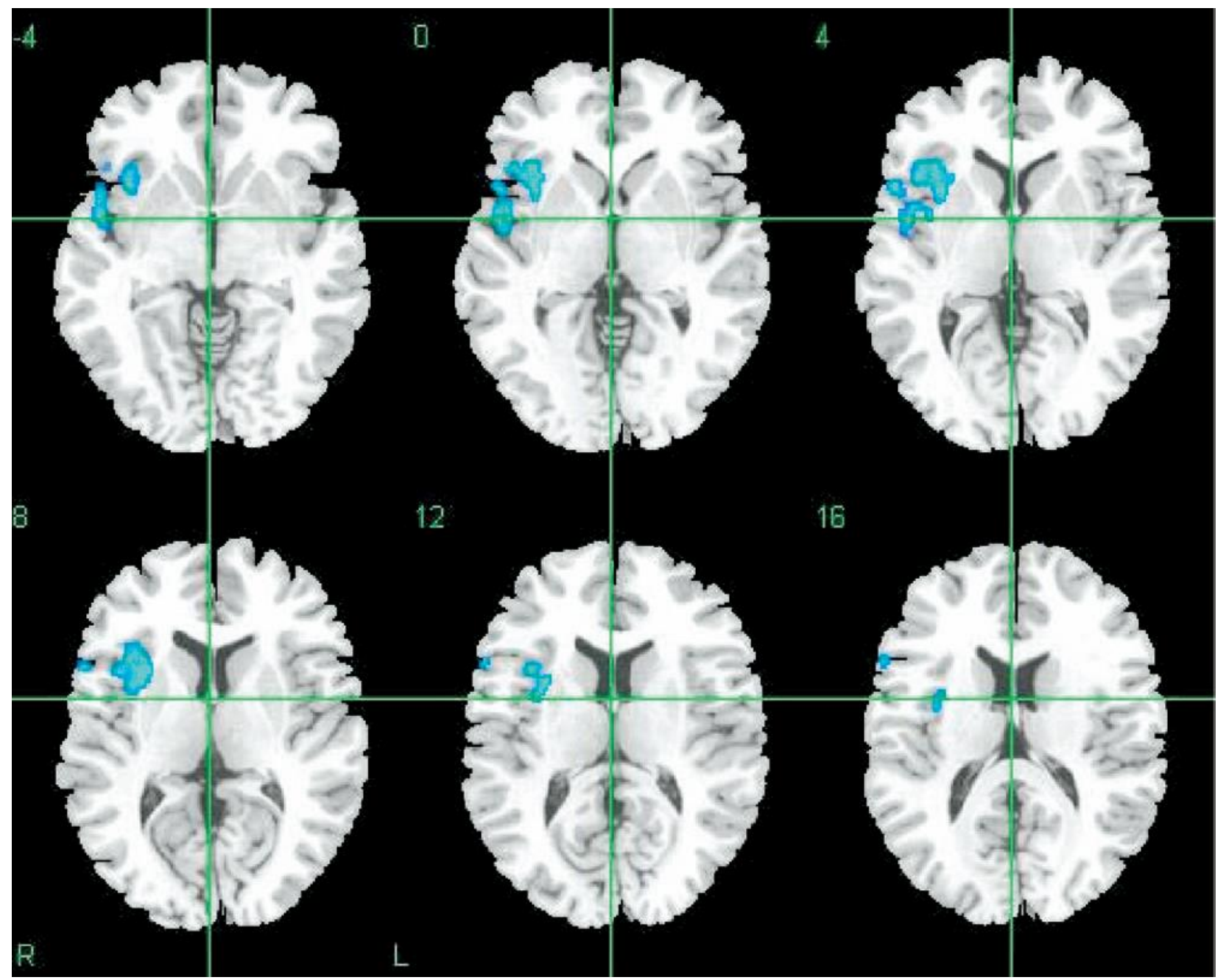

Reprinted from Biological Psychiatry, Vol. 61, Borgwardt SJ, Riecher-Rössler A, Dazzan P, et al. Regional gray matter volume abnormalities in the at risk mental state, pp. 1148-1156, 2007, with permission from Elsevier. 